\title{
Game-based data offloading scheme for loT system traffic congestion problems
}

\author{
Youngjae Park and Sungwook Kim ${ }^{*}$
}

\begin{abstract}
Internet of things $(\mathrm{I} O T)$ is seen as another information and industrial wave after the invention of personal computers, the Internet, and mobile communication networks. Especially, loT/cellular network integration becomes a new service platform for the different kinds of traffic manipulation. However, due to the excessive traffic demands, it is currently facing a severe traffic overload problem. In this paper, we propose a new traffic control scheme based on the data offloading technique. By using the Vickrey-Clarke-Groves (VCG) mechanism and Rubinstein bargaining game model, our data offloading approach can effectively alleviate the loT traffic congestion while enhancing the quality-of-service (QoS) in cellular network systems. Finally, we show the effectiveness of our proposed scheme through extensive simulations.
\end{abstract}

Keywords: Internet of things; Data offloading; Game theory; VCG mechanism; Rubinstein bargaining model

\section{Introduction}

Internet of things (IoT) is a novel paradigm that integrates several technologies such as wired and wireless networks, enhanced communication protocols, distributed intelligence for smart objects, mobile phones, and undoubtedly the Internet. The basic idea of IoT is to connect things to enhance several aspects of everyday life and behavior of potential users. Especially, with cellular network systems, ubiquitous public services would be of enormous benefit. However, due to the unprecedented worldwide growth of data traffic, which is expected to reach 10.8 exabytes per month by 2016 , an 18-fold increase over 2011, effective traffic congestion mechanisms are needed [1-4]. To cope with explosive traffic demands, traditional network expansion methods have been developed while acquiring more spectrum licenses, deploying new micro-cells of small size, and upgrading technologies. But, these approaches are costly and time-consuming. Therefore, network operators must find a new novel method to resolve the mismatch between network capacity and traffic growth $[2,5,6]$.

\footnotetext{
* Correspondence: swkim01@sogang.ac.kr

Department of Computer Science, Sogang University, 35 Baekbeom-ro (Sinsu-dong), Mapo-gu, Seoul 121-742, South Korea
}

For IoT/cellular network systems, mobile data offloading appears as one of the most attractive data delivering solutions. Data offloading mechanism has been developed to offload traffic from cellular networks to high capacity and free device-to-device networks. Recently, a growing number of studies have been devoted to the potential performance benefits of mobile data offloading and the technologies to support it. However, the existing researches only focused on the technical aspect of data offloading without considering the economic incentive for access point owners (APOs) to admit cellular network traffic. This incentive issue is particularly important for the scenario where APOs are privately owned by third-party entities who are expected to be reluctant to admit non-registered users' traffic without proper incentives [2].

In this paper, we consider a new user-initiated data offloading scheme. In the proposed scheme, Internet of things modules (IoTMs) initiate the offloading process and the mobile network operators (MNOs) are responsible of incentivizing APOs. As users, IoTMs are assumed either (1) compliant or properly incentivized, such that they will offload their traffic exactly as the networks intended or (2) unaware of the offloading process at all; it means that data offloading is totally transparent to IoTMs. In this work, data offloading actions are only triggered by either an IoTM or a MNO. To reduce

\section{实 Springer}


communication costs, IoTMs offload data while the MNO tries to ease traffic congestion of cellular networks. Basically, we can assume that IoTMs, MNO, and APOs can make strategic decisions independently and rationally to maximize their payoffs. Due to this reason, game theory can be a suitable approach for data offloading scenarios.

Game theory is a field of applied mathematics that provides an effective tool in modeling the interactions among independent decision-makers. It can describe the possibility to react to the actions of the other decisionmakers and analyze the situations of conflict and cooperation [7]. In this paper, we adopt Vickrey-Clarke-Groves (VCG) mechanism and Rubinstein bargaining game model to design the data offloading algorithm. The VCG mechanism implements efficient social choice functions in environments in which participants have private information about their preferences. In this mechanism, the game participants' payoffs equal their respective marginal contributions to the social surplus [8]. A Rubinstein bargaining model refers to a class of bargaining games that feature alternating offers through an infinite time horizon. Rubinstein's solution is one of the most influential findings in game theory [9]. Based on the combination of VCG mechanism and Rubinstein bargaining model, MNO, APOs, and IoTMs participate in the data offloading process for the effectiveness of IoT/cellular system operation.

The main contributions of our work are as follows:

1. We propose a new pricing method by considering the characteristics of APOs and IoTMs. This method can induce the cooperation between APOs and IoTMs in data offloading operations.

2. Based on the Rubinstein bargaining model, we can fairly distribute the surplus profit. It can ensure the system effectiveness.

3. Through the adaptive combination of VCG mechanism and Rubinstein bargaining model, MNO, APOs, and IoTMs actively participate in the data offloading process in the IoT/cellular system environment.

The rest of the paper is structured as follows. In Section 2, we survey previous work in resource allocations, auction-based game theory models, and data offloading algorithms. In Section 3, we explain in detail our proposed mobile data offloading algorithm. In Section 4, we discuss the experimental environment for performance evaluation and analyze simulation results. Finally, we conclude the paper in Section 5.

\section{Related work}

In this chapter, we review the recently researched literatures that relate to our proposed scheme. Currently, several resource allocation schemes have been deployed for wireless networks. Byun et al. [10] have investigated a market equilibrium in multichannel sharing cognitive radio networks. They model the spectrum-sharing process as a spectrum market where primary users offer secondary users their subchannels with limiting the interference from secondary users. And then, they define a market equilibrium in the context of extended Fisher model and show that the equilibrium is yielded by solving an optimization problem, Eisenberg-Gale convex program. Finally, they develop a distributed algorithm that yields the stationary solutions asymptotically equivalent to the solutions given by the convex program [10].

Jiang et al. [11] have proposed a novel quality-ofexperience (QoE)-driven channel allocation scheme for secondary users and cognitive radio networks base station. The historical QoE data under different primary channels are collected by the secondary users and delivered to a cognitive radio base station, which will allocate available channel resources to the secondary users based on their QoE expectations and maintain a priority service queue. The modified ON/OFF models of primary channels and service queue models of secondary users are jointly investigated for this channel allocation scheme [11].

In [12], Zhang et al. have conducted the first work on a framework for truthful online cloud auctions where users with heterogeneous demands could come and leave on the fly. Their framework desirably supports a variety of design requirements, including (1) dynamic design for timely reflecting fluctuation of supply-demand relations, (2) joint design for supporting the heterogeneous user demands, and (3) truthful design for discouraging bidders from cheating behaviors. Their theoretical analysis shows that the worst-case performance of their mechanism can be well-bounded [12].

Feng et al. [13] tackled the problem of stimulating smartphone users to join mobile crowdsourcing applications with smartphones. They have introduced a reverse auction framework to model the interactions between the platform and the smartphones and have proposed a mechanism called TRAC which consists of two main components. The first component is a near-optimal approximate algorithm for determining the winning bids with polynomial-time computation complexity. The second component is a critical payment scheme which, despite the approximation of determining winning bids, guarantees that submitted bids of smartphones reflect their real costs of performing sensing tasks [13].

In [14], Rahimi et al. have exploited the observation that using local resources in close proximity to the user can increase the quality and performance of mobile applications. In this paper, they introduced MAPCloud, a 
hybrid, tiered cloud architecture consisting of local and public clouds, and showed how it can be leveraged to increase both performance and scalability of mobile applications. They modeled the mobile application as a workflow of tasks and aim to optimally decompose the set of tasks to execute on the mobile client and two-tier cloud architecture considering multiple quality-of-service (QoS) factors such as power, price, and delay. Their results indicated that MAPCloud can provide improved scalability and efficiency in comparison to only using public cloud [14].

In the paper [15], authors have proposed a novel framework to model mobile applications as a locationtime workflows of tasks and showed that an optimal mapping of location-time workflows to tiered mobile cloud resources is an NP-hard problem. In addition, they proposed an efficient heuristic algorithm that can perform well and scale well to a large number of users while ensuring high application QoS. They also showed that their proposed scheme can perform quite well under different mobility patterns and be resilient to errors/ uncertainty in prediction of mobile user location-time workflows [15].

The paper [16] discussed the current state of the art in the merger of cloud computing and smartphone technologies that is called as mobile cloud computing (MCC). This paper also illustrated the applicability of MCC in various domains including mobile learning, commerce, health/wellness, and social media. In addition, this research would identify research gaps covering critical aspects of how MCC can be realized and effectively utilized at scale. These include improved resource allocation in the MCC environment through efficient task distribution and offloading, security, and privacy [16].

$\mathrm{Xu}$ et al. [17] have reviewed the state-of-the-art research on managing the performance overhead of virtual machines and summarize them under diverse scenarios of the infrastructure-as-a-service (IaaS), ranging from the single-server virtualization, a single mega datacenter, to multiple geodistributed datacenters. Specifically, they unveiled the causes of virtual machine performance overhead by illustrating representative scenarios, discussed the performance modeling methods with a particular focus on their accuracy and cost, and compared the overhead mitigation techniques by identifying their effectiveness and implementation complexity. Finally, they presented future research challenges pertinent to the modeling methods and mitigation techniques of virtual machine performance overhead in the IaaS cloud [17].

Demestichas et al. [18] identified and addressed the important problem of service configuration and distribution in a composite radio environment (SCD-CRE).
The problem's objective was to determine the best way to accommodate a demand volume, with which the composite radio infrastructure was faced. They found the QoS levels that can be offered and the networks that can support the demand at the selected QoS levels. In addition, they included a first approach to the definition, mathematical formulation, and solution of a version of the SCD-CRE problem. Results indicating some of the capabilities of the SCD-CRE were also presented [18].

Yan et al. [19] investigated the properties of trust, proposed objectives of the IoT trust management, and provided a survey on the current literature advances towards trustworthy IoT. Furthermore, they discussed unsolved issues, specified research challenges and indicated future research trends by proposing a research model for holistic trust management in IoT. In addition, they explored the literature towards trust worthy IoT in order to point out a number of open issues and challenges and suggested future research trends related to trust management. Finally, they presented a further research model in order to achieve comprehensive trust management in IoT [19].

To deal with the problem of cellular traffic overload, some methods have been studied to efficiently conduct offloading. Zhuo et al. considered the use of the VCG model for data offloading [8]. This model investigates the tradeoff between the amount of traffic being offloaded and the users' satisfaction and provides a novel incentive framework to motivate users to leverage their delay tolerance for cellular traffic offloading. To minimize the incentive cost given an offloading target, users with high delay tolerance and large offloading potential should be prioritized for traffic offloading. To effectively capture the dynamic characteristics of users' delay tolerance, the scheme in [8] lets users proactively express their delay tolerance based on the reverse auction. Gao et al. considered the use of the or Nash Bargaining Solution (NBS) in the data offloading system [20]. In this model, the MNO and APOs analyze the interaction among one MNO and multiple APOs based on the Nash bargaining theory.

\section{The proposed data offloading algorithm}

First, we design a game model of mobile data offloading system and then explain the proposed algorithm in detail. In this work, the data offloading system distributes IoTM's traffic. The three categories of the players are MNO, APOs, and IoTMs. We denote a set of IoTMs as $\mathcal{N}$, such that $\mathcal{N}=\{1,2, \ldots, N\}$. Each IoTM has a traffic that wants to offload their cellular traffic. And a set $\mathcal{K}=\left\{\mathrm{APO}_{1}, \mathrm{APO}_{2}, \ldots, \mathrm{APO}_{K}\right\}$ consists of third-party Wi-Fi or femtocell APOs. Each $\mathrm{APO}_{i, 1 \leq i \leq K}$ has a set $\mathscr{M}_{i}=\left\{1_{i}, 2_{i}, \ldots, M_{i}\right\}$ of available 
channels and each $\mathrm{APO}_{i}$ has covered a number of IoTMs $\left(\operatorname{APO}_{i}(\mathcal{N})\right)$ where $\operatorname{APO}_{i}(\mathcal{N}) \subset \mathcal{N}$. Based on this topology, we develop two game models-VCG mechanism and Rubinstein game-to manage the data offloading in IoT systems.

We consider an effective incentive model for the userinitiated data offloading. In our model, IoTMs initiate the offloading process while offering necessary incentives in order to access to APs. Generally, IoTMs should pay the price for their traffic transmission to a mobile network operator (MNO). Usually, a huge amount of cellular data traffic has been generated by IoTMs, which exceeds the capacity of cellular network. It deteriorates the network quality. Therefore, the MNO should offload the part of cellular traffic to other coexisting networks. This technique is a desirable and promising approach to solve the cellular network overload problem. To motivate IoTMs and APOs to help cellular traffic offloading, we design an incentive mechanism by considering the IoTMs' delay tolerance and price discount factor. In our model, IoTMs can reduce their payment by using an APO bandwidth, which can be used for the IoTMs' cellular traffic services at a discounted price. On the other hand, APOs can get a surplus profit through providing their remaining bandwidth. To formalize MNO, APOs, and IoTMs actions, we develop a two-level game model based on VCG mechanism and Rubinstein bargaining solution. Under dynamic IoT changing situations, our game-based approach can toward an effective system performance (Fig. 1).

\subsection{VCG mechanism for cooperation between APO and loTMs}

From the viewpoint of APOs, channel allocation to IoTMs is a very important. To maximize their profit, APOs try to select the most adaptable IoTM, which has more offloading traffic and higher signal-to-interferenceplus-noise ratio (SINR). In this paper, we adopt the VCG mechanism to address the channel allocation problem among IoTMs. In our VCG mechanism model, $\mathrm{APO}_{i}$ can be assumed as a seller with $M_{i}$ channels, and each channel is allocated to a specific IoTM. For simplicity, we assume that the transmission range of APO is much smaller than that of the MNO and the coverage areas of any two APOs are not overlapped. For data offloading, each IoTM wants to obtain channels from its corresponding APO. With a discounted price, IoTMs enjoy an additional profit for data transmission in a time slot. However, APO's channels are not enough to meet the total data offloading requests from IoTMs. Therefore, as a buyer, each IoTM bid for getting channels from its corresponding APO. To solve this channel allocation problem, our game model $\mathbb{G}=\left\{\mathcal{N}, \mathcal{K}, \mathbb{S}_{\mathrm{IoTM}}, \mathbb{S}_{\mathrm{APO}}, \boldsymbol{U}_{\mathrm{APO}}, \boldsymbol{U}_{\mathrm{IoTM}}\right\}$ is defined as follows.
- Players $(\mathcal{N}, \mathcal{K}): \mathcal{N}$ and $\mathcal{K}$ are set of APOs and set of IoTMs, respectively, and the IoTMs in the coverage of $\mathrm{APO}_{i}$ is $\mathrm{APO}_{i}\left(\mathcal{N}_{i}\right)$.

- Strategies $\left(\mathbb{S}_{\text {IoTM }}, \mathbb{S}_{\mathrm{APO}_{i}}\right)$ : $\mathbb{S}_{\text {IoTM }}$ is the strategy set for each IoTM and $\mathbb{S}_{\mathrm{APO}_{i}}$ is the strategy set for $\mathrm{APO}_{i}$. $\mathbb{S}_{\text {IoTM }}$ is IoTM's bid strategy set and $\mathbb{S}_{\mathrm{APO}_{i}}$ is the channel allocation vector $\left(A_{i}\right)$.

- Payoff functions $\left(U_{\mathrm{APO}_{i}}(\cdot), U_{\mathrm{IoTM}}(\cdot)\right): U_{\mathrm{APO}_{i}}$ and $U_{\text {IoTM }}$ are the payoff functions of $\mathbb{S}_{\text {IoTM }}$ and $\mathbb{S}_{\mathrm{APO}_{i}}$, respectively.

$\boldsymbol{A}_{i}$ is the strategy of $\mathrm{APO}_{i}$ where $i \in \mathcal{K}$, its channel allocation vector. $b_{n_{i}}$ is the strategy of $n_{i}$ where $n_{i} \in \mathrm{APO}_{i}$ $(\mathcal{N})$; it is the bid to get the channel. All the players try to maximize their payoff. The $n_{i}$ 's payoff function ( $\left.U_{\mathrm{IoTM}_{n_{i}}}\left(\boldsymbol{b}_{\boldsymbol{i}}\right)\right)$ for surplus benefit is defined with the offloading data traffic and discounted rate $\left(P_{n_{i}}\right)$.

$$
U_{\mathrm{IoTM}_{n}}\left(n_{i}, \boldsymbol{b}_{i}\right)=P_{n_{i}} \times d_{n_{i}}
$$

where $d_{n_{i}}$ is the amount of $n_{i}$ 's data offloading traffic. The $\boldsymbol{b}_{\boldsymbol{i}}=\left\{b_{1_{i}}, b_{2_{i}}, . ., b_{N_{i}}\right\}$ is the vector of received bids from IoTMs, and each $b_{n_{i}} \in \boldsymbol{b}_{\boldsymbol{i}}$ is decided according to the $n_{i}$ 's SINR and $d_{n_{i}}$.

$$
\begin{aligned}
b_{n_{i}} & =\left\{\operatorname{SINR}_{n_{i}}, d_{n_{i}}\right\} \text { s.t., } \operatorname{SINR}_{n_{i}} \\
& =\left\{\operatorname{SINR}_{n_{i}}^{1}, \operatorname{SINR}_{n_{i}}^{2}, \ldots, \operatorname{SINR}_{n_{i}}^{m}\right\} \text { and } \operatorname{SINR}_{n_{i}}^{m}=\frac{\mathcal{P}_{n_{i}} h r^{-\alpha}}{\sigma^{2}+I_{r, m}}
\end{aligned}
$$

where $\mathcal{P}_{n_{i}}$ is the transmit power of $n_{i}$ and $h r^{-\alpha}$ is the distance between $\mathrm{APO}_{i}$ and $n_{i} . m_{i}^{j}$ is an available channel of $\mathrm{APO}_{i}$, i.e., $m_{i}^{j}=\left\{1,2, \ldots, m_{i}\right\} \in \mathscr{M}_{i}$. $\sigma^{2}$ and $I_{r, m}$ are the noise power and interference power, respectively [21]. $\mathrm{APO}_{i}$ 's payoff function $\left(U_{\mathrm{APO}_{i}}\left(\boldsymbol{A}_{i}\right)\right)$ means the expected payoff when $\mathrm{APO}_{i}$ selects $\boldsymbol{A}_{i}$ channel allocation. In this work, this function is developed based on the Rubinstein bargaining game, which is explained in detail in the Subsection 3.2.

Finally, we develop a new a social welfare function to represent a total system efficiency; it can be estimated through an effective data offloading technique. In this work, channel allocation is operated by each APO. After receiving all bids $\boldsymbol{b}_{\boldsymbol{i}}=\left(b_{1_{i}}, b_{2_{i}}, . ., b_{N_{i}}\right), \mathrm{APO}_{i}$ decides the channel allocation vector $\boldsymbol{A}_{i}=\left(\boldsymbol{a}_{1 i}, \boldsymbol{a}_{2 i} \ldots \boldsymbol{a}_{n i}\right)$ as follows:

$$
\begin{aligned}
& \max _{\boldsymbol{A}_{\boldsymbol{i}}^{*}} U_{\mathrm{APO}_{i}}\left(\boldsymbol{A}_{\boldsymbol{i}}, \boldsymbol{b}_{\boldsymbol{i}}\right)=\sum_{n_{i}=1}^{N_{i}} \sum_{j=1}^{M_{i}} \operatorname{SINR}_{n_{i}}^{j} \times d_{n i} \times a_{n_{i}}^{j} \\
& \text { s.t. }\left\{\begin{array}{l}
\left\{\operatorname{SINR}_{n_{i}}^{j}, d_{n_{i}}\right\} \subset b_{n_{i}} \in \boldsymbol{b}_{\boldsymbol{i}}, \\
\boldsymbol{A}_{\boldsymbol{i}}=\left(\boldsymbol{a}_{1}, \boldsymbol{a}_{2}, \ldots, \boldsymbol{a}_{N_{i}}\right), \boldsymbol{a}_{n_{i}} \\
=\left(a_{n_{i}}, a_{n_{i}}, \ldots, a_{n_{i}}{ }^{M_{i}}\right), \boldsymbol{a}_{n_{i}} \in\{0,1\}
\end{array}\right.
\end{aligned}
$$




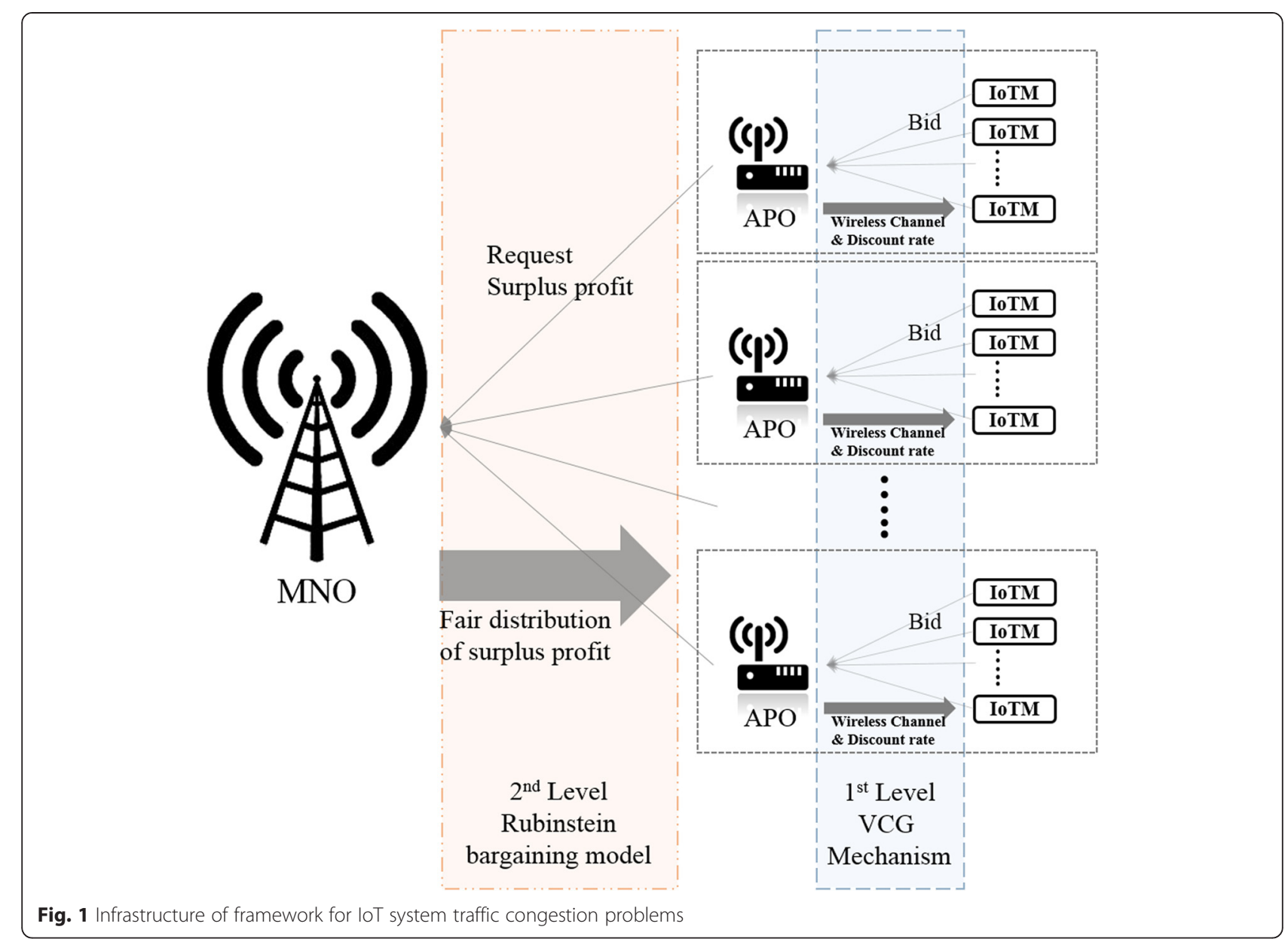

where $a_{n i} \in\{0,1\}$ is the indicator factor whether the $n_{i}$ gets a channel $\left(a_{n i}=1\right)$ or not $\left(a_{n i}=0\right)$.

From the IoTM $\left(n_{i}\right)$ 's point of view, the discounted rate $\left(P_{n_{i}}\right)$ for data transmission is the main interest; it is obtained through data offloading. In the proposed model, we estimate the $P_{n_{i}}$ value based on the VCG mechanism. The basic idea of VCG mechanism is a second-price auction. Among multiple bidders, the bidder who has the highest bid wins the auction, but the winner only pays the social opportunity cost, which means the different payoff that total APO's payoff that would be gotten with or without the winner's payoff. In the proposed scheme, the social opportunity $\operatorname{cost}\left(S_{C_{n_{i}}}\right)$ is given by.

$$
S_{-} C_{n_{i}}=U_{\mathrm{APO}_{i}}\left(\boldsymbol{A}_{\boldsymbol{i}_{-n}}^{*}\right)-U\left(\boldsymbol{A}_{\boldsymbol{i}}^{*}\right)
$$

where $A_{i_{n}}^{*}$ is the optimal channel allocation vector without $n$th IoTM. We assume that an individual social opportunity cost is each IoTM's contribution to the total network system. Therefore, the discount rate
$P_{n_{i}}$ is defined as the ratio of the social opportunity costs.

$$
P_{n_{i}}=-\frac{S_{-} C_{n_{i}}}{\sum_{j \in \mathcal{N}_{i}} S_{-} C_{j}}
$$

\subsection{Rubinstein bargaining model for distribution of surplus profit}

The main issue between the MNO and APOs is to maximize the profit by cooperation and fairly divide the surplus profit. To satisfy this goal, we propose a new fair division model based on the Rubinstein-Stahl bargaining approach. In our game model, the MNO negotiates with each APO, sequentially. Through several rounds of offers and counteroffers, they finally come to an agreement. From the APOs' point of view, they try to maximize their profit by providing their remaining bandwidth. On the other hand, the major goal of the MNO is to fairly share this surplus profit getting from the bandwidth sharing. To address this division problem involving two players, Rubinstein bargaining game can provide a unique solution to fairly share a given benefit [9]. 
The MNO's surplus profit $\left(W_{i}\right)$, which is obtained through $\mathrm{APO}_{i}$ 's data offloading, is determined as the difference between the MNO's cost with and without data offloading. First, the MNO's cost function without data offloading $(\mathrm{C}(\mathrm{MNO}))$ is given by

$$
\mathrm{C}(\mathrm{MNO})=\varepsilon *\left(F_{\text {total }}\right)^{2} \text {, s.t., } F_{\text {total }}=\sum_{n \in \mathcal{N}} z_{n}+\sum_{i \in \mathcal{K}} \sum_{m \in \mathscr{M}_{i}} d_{m}^{i}
$$

where $F_{\text {total }}$ is the total traffic of MNO. $z_{n}$ is the traffic amount serviced by MNO, and $d_{m}^{i}$ is the traffic amount serviced by the channel $m$ in $\mathrm{APO}_{i}$. In this work, we follow the common sense; a generic cost function $C(x)$ is considered as continuous, differentiable, strictly increasing, and convex [2]. Therefore, the MNO's cost function with data offloading $(\mathrm{D}(\mathrm{MNO}))$ is calculated as follows:

$$
\begin{aligned}
& \mathrm{D}(\mathrm{MNO})=\mathrm{C}\left(F_{D_{-} O}\right)-\left(\sum_{m \in \mathscr{M}_{i}} \mathrm{P}\left(1-P_{m}^{i}\right) \times d_{m}^{i}\right), \text { s.t. }, \\
& F_{D_{O}}=F_{\text {total }}-\sum_{m \in \mathscr{M}_{i}} d_{m}^{i}
\end{aligned}
$$

where $F_{D} O$ is the total traffic of $\mathrm{MNO}$ with data offloading. The second factor of the Eq. 5 is the discount cost caused by data offloading; $\mathrm{P}$ and $P_{m}^{i}$ are the cellular network base price and the discount rate of channel $m$ in $\mathrm{APO}_{i}$, respectively. Finally, the MNO's surplus profit $\left(W_{i}\right)$ is given by:

$$
W_{i}=\mathrm{C}(\mathrm{MNO})-\mathrm{C}\left(F_{D_{0}}\right)-\left(\sum_{m \in \mathscr{M}_{i}} P\left(1-P_{m}^{i}\right) \times d_{m}^{i}\right)
$$

In the Rubinstein bargaining model, final agreement can be expressed as follows:

$$
\begin{aligned}
& \left(x_{\mathrm{APO}_{i}}^{*}, x_{\mathrm{MNO}}^{*}\right)=\left(\frac{1-\delta_{\mathrm{MNO}}}{1-\delta_{\mathrm{APO}_{i}} \delta_{\mathrm{MNO}}}, \frac{\delta_{\mathrm{MNO}}\left(1-\delta_{\mathrm{APO}_{i}}\right)}{1-\delta_{\mathrm{APO}_{i}} \delta_{\mathrm{MNO}}}\right) \\
& \times \text { s.t. }\left(x_{\mathrm{APO}_{i}}^{*}, x_{\mathrm{MNO}}^{*}\right) \in \boldsymbol{R}^{2}: x_{\mathrm{APO}_{i}}^{*} \\
& +x_{\mathrm{MNO}}^{*} \\
& =1 x_{\mathrm{APO}_{i}}^{*} \geq 0, x_{\mathrm{MNO}}^{*} \geq 0 \text {, and } 0 \leq \delta_{\mathrm{MNO}}, \delta_{\mathrm{APO}_{i}} \leq 1
\end{aligned}
$$

Let $\delta_{\mathrm{APO}_{i}}$ and $\delta_{\mathrm{MNO}}$ be the respective $\mathrm{MNO}$ and $\mathrm{APO}_{i}$ patience factors. In the Rubinstein bargaining process, the more patience one has, the more payoff one attains. Under the common scenario of data offloading process, APOs have the higher precedence than MNO; after $\mathrm{APO}_{i}$ 's data offloading decisions, $\mathrm{MNO}$ can plan to effectively allocate its resource to obtain the most profitable usage. Due to this reason, we assume that $\mathrm{APO}_{i}$ has greater patience than MNO's patience. In this work, we can represent MNO's patience as a monotonic time- decreasing function and $\mathrm{APO}_{i}$ 's patience as a monotonic time-increasing function as follows [9]:

$$
\left\{\begin{aligned}
\delta_{\mathrm{MNO}}(r)=1-\frac{\mathrm{e}^{\pi_{i}^{r}}-\mathrm{e}^{-\pi_{i}^{r}}}{\mathrm{e}^{\pi_{i}^{r}}+\mathrm{e}^{-\pi_{i}^{r}}} & \\
\text { s.t. } \frac{\mathrm{d} \delta_{\mathrm{MNO}}(r)}{\mathrm{d} r}<0, & \delta_{\mathrm{MNO}}(0)=1 \text { and } \\
\delta_{\mathrm{APO}_{i}}(r)=\frac{e^{\pi_{i}^{r}}-e^{-\pi_{i}^{r}}}{e^{\pi_{i}^{r}}+e^{-\pi_{i}^{r}}} & \\
\text { s.t. } \frac{d \delta_{\mathrm{APO}_{i}}(r)}{d r}>0, & \delta_{\mathrm{APO}_{i}}(0)=0, \text { and } \\
& \delta_{\mathrm{APO}_{i}}(\infty)=1
\end{aligned}\right.
$$

where $\pi_{i}^{r}$ is the $i$ th APO's patience coefficient in the $r$ th bargaining iteration round. The patience coefficient $\left(\pi_{i}^{r}\right)$ strongly affects the patience factor of both sides. Therefore, for the ideal management of data offloading system, we dynamically adjust the value of $\pi_{i}^{r}$. To maximize the social welfare, $\pi_{i}^{r}$ can be defined as follows:

$$
\begin{gathered}
\pi_{k}^{r}=\frac{\mathrm{C}\left(\sum_{k \in \mathcal{K}} \sum_{m \in \mathscr{M}_{k}} d_{m}^{k}\right)-C\left(\sum_{l \in \mathcal{K}_{-k}} \sum_{m \in \mathscr{M}_{l}} d_{m}^{k}\right)}{\mathrm{C}\left(\sum_{k \in \mathcal{K}} \sum_{m \in \mathscr{M}_{k}} d_{m}^{k}\right)} \\
\text { s.t., } \sum_{l \in \mathcal{K}_{-i}} \sum_{m \in \mathscr{M}_{l}} d_{m}^{l}<\sum_{i \in \mathcal{K}} \sum_{m \in \mathscr{M}_{i}} d_{m}^{i}<F_{\text {total }}
\end{gathered}
$$

where $\mathcal{K}_{-i}$ represents the all APOs except $\mathrm{APO}_{i}$. The denominator in the Eq. 11 is the total cost $\left(\mathrm{C}\left(\sum_{k \in \mathcal{K}} \sum_{m \in \mathscr{A}_{k}} d_{m}^{k}\right)\right)$, and the numerator is the difference of the social welfare with the $\operatorname{APO}_{i}\left(\mathrm{C}\left(\sum_{i \in \mathcal{K}} \sum_{m \in \mathscr{M}_{i}} d_{m}^{i}\right)\right)$ and without the $\operatorname{APO}_{i}\left(C\left(\sum_{l \in \mathcal{K}_{-} m \in \mathcal{M}_{l}} \sum_{m}^{l}\right)\right)$. Finally, we can define the weighted factor according to Eqs. 9, 10, and 11.

$$
\omega=\frac{1-\delta_{\mathrm{MNO}}}{1-\delta_{\mathrm{APO}_{i}} \delta_{\mathrm{MNO}}} \text { and }(1-\omega)=\frac{\delta_{\mathrm{MNO}}\left(1-\delta_{\mathrm{APO}_{i}}\right)}{1-\delta_{\mathrm{APO}_{i}} \delta_{\mathrm{MNO}}}
$$

According to Eq. 12, MNO and APOs can get a surplus profit as follows.

$$
R_{\mathrm{APO}_{i}}=\omega \times W_{i} \quad \text { and } \quad R_{\mathrm{MNO}}=(1-\omega) \times W_{i}
$$

where $R_{\mathrm{APO}_{i}}$ is the surplus profit for $\mathrm{APO}_{i}$ and $R_{\mathrm{MNO}}$ is the surplus profit for MNO.

\subsection{The main steps of proposed algorithms}

In this paper, we develop a new cooperative data offloading scheme based on the VCG mechanism and Rubinstein bargaining approach. In the proposed scheme, APOs and IoTMs are game players and participate in the data offloading game to maximize their payoffs. During the iterative game process, IoTMs can take pleasure in the discounted price of cellular service and APOs can get a surplus profit. The main steps of 


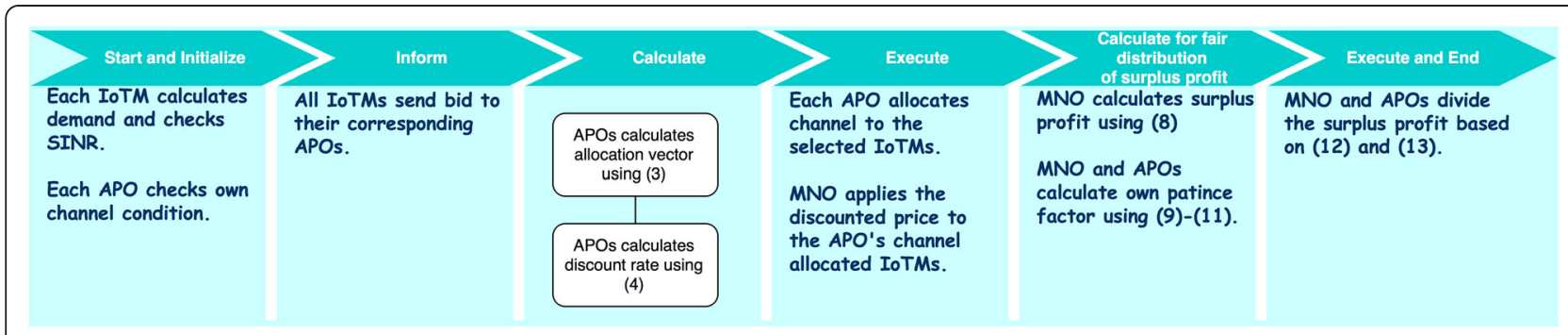

Fig. 2 Flow chart of proposed scheme

the proposed data offloading scheme are given next (Fig. 2) (Algorithm 1).

Step 1: At the initial time, each IoTM calculates the amount of data offloading traffic and the SINR. At the same time, each APO checks its own channel condition.

Step 2: All IoTMs send bid message $b_{n}$ to their corresponding APOs.

Step 3: Each APO determines the optimal IoTMs and calculates the discount rate using Eqs. 3, 4, and 5.
Step 4: Each APO allocates channels to the selected IoTMs and the MNO applies the discounted price to the APO's channel allocated IoTMs.

Step 5: After the channel allocation, the MNO should calculate the surplus profit using Eq. 8.

Step 6: According to Eqs. 9-11, the MNO and APOs calculate their own patience factors.

Step 7: Finally, the MNO and APOs divide the surplus profit based on Eqs. 12 and 13.

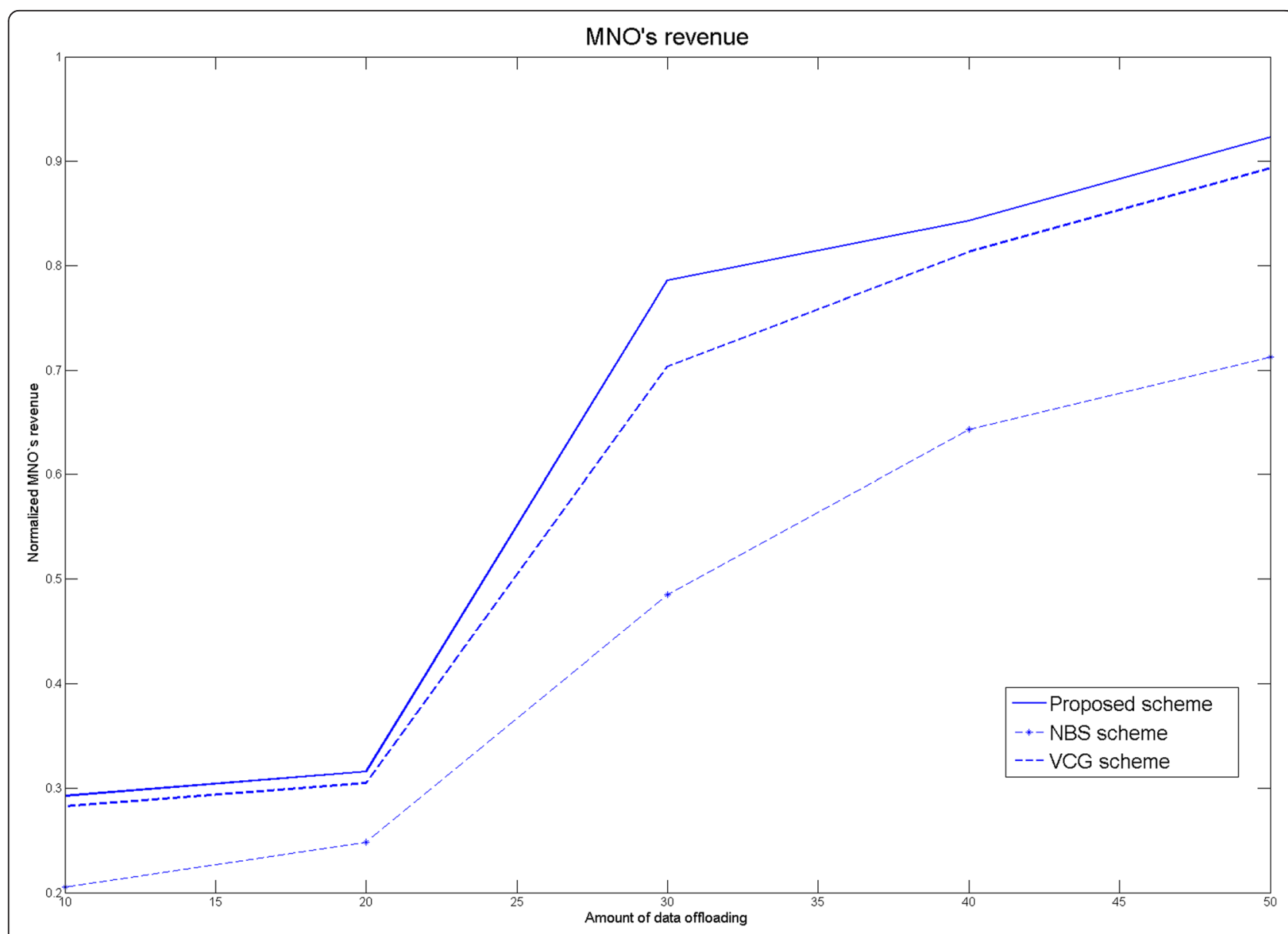

Fig. 3 MNO's revenue 


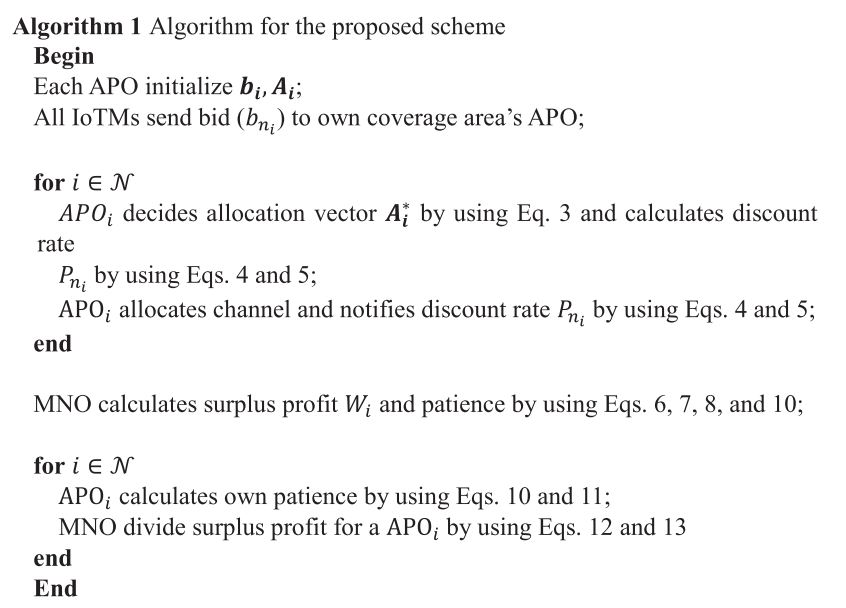

\section{Performance evaluation}

In this section, the effectiveness of the proposed scheme is validated through simulation. Using a simulation model, which emulates a real-world IoT data offloading environment, the performance of the proposed scheme is compared with the two existing data offloading management schemes; the VCG scheme [9] and the NBS scheme [20]. The assumptions implemented in simulation model are as follows.

- Simulation system performance measures obtained on the basis of 50 iterations.

- There are ten APOs and each APO has ten IoTMs that can be covered by APO.

- Wi-Fi APOs are typical 3G/4G micro-cells with the 50-m transmission range.

- The APOs are deployed in the high IoTM density areas.

- Each IoTM's traffic is a randomly and uniformly selected from 0 to $10 \mathrm{Mbps}$.

- Performance criteria obtained through simulations are the MNO's revenue, the APOs' revenue, and the IoTMs' discount rate of price.

- MNO's revenue is estimated as the decreasing rate of MNO's cost; it is caused by the data offloading technique.

- APOs' revenue is defined as the profit, which is obtained from the MNO.

- IoTMs' discount rate represents the ratio of the discount price to the original price.

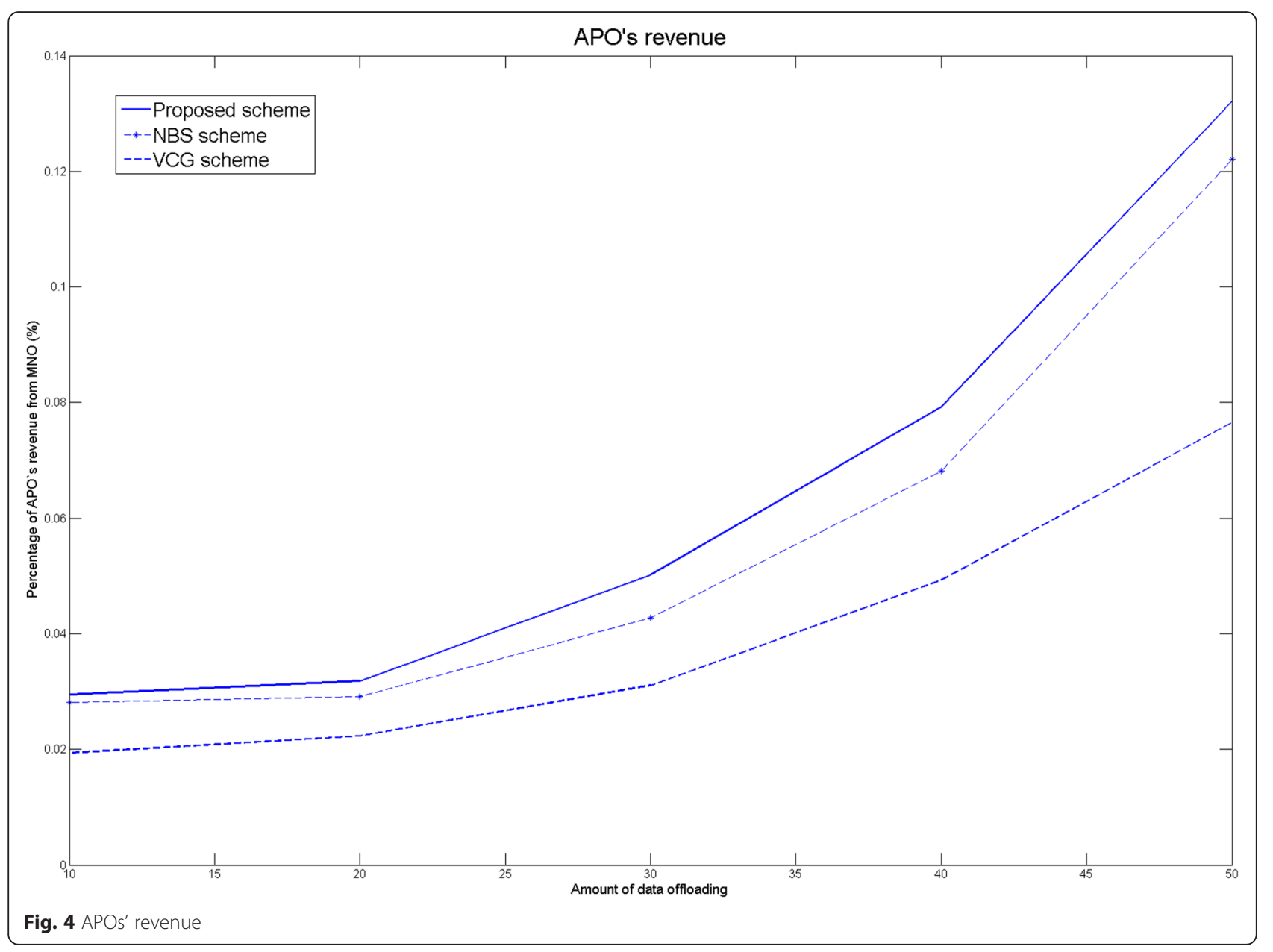


In Fig. 3, the MNO's revenues of each scheme are compared over different data offloading traffics. From the simulation results, we can see that the MNO's revenue of our proposed scheme has higher revenue than those of other existing schemes. Based on these considerations, APOs and IoTMs, it shows that our scheme can effectively decrease the MNO's cost more than other schemes.

Figure 4 shows the performance comparison about the aggregate percentage of APOs' revenue, which represents the APOs' contribution for the data offloading. In this work, it is obtained from the MNO's surplus profit. From simulation results, the main observation is that the APOs' revenue of our scheme is better than the other existing schemes.

Figure 5 shows the IoTMs' average percentage of discount rate. Usually, a higher discount rate value means that IoM users can reduce their payment. In our scheme, IoTMs and APOs actively participate in the data offloading game process and decrease the cost of data transmission. In addition, we can effectively allocate the APO's bandwidth for data offloading. For this reason, IoTM users in our scheme tend to have a significantly lower payment than other existing schemes while improving the efficiency of an IoT-data-offloading system.

From the simulation results in Figs. 3, 4, and 5, it can be seen that our proposed approach performs better than the VCG scheme [8] and the NBS scheme [20]. Based on the combination of VCG mechanism and Rubinstein bargaining approach, the proposed scheme can constantly monitor the IoT system conditions and appropriately balance the system performance, whereas the other schemes $[8,20]$ cannot offer such an attractive system performance.

\section{Conclusions}

Recently, the explosive growth of multimedia traffic services has necessitated to design an efficient data offloading algorithm for the IoT/cellular network system. Data offloading is a promising technique to alleviate network traffic congestion and enhance service QoS. In this paper, we develop a new data offloading algorithm based

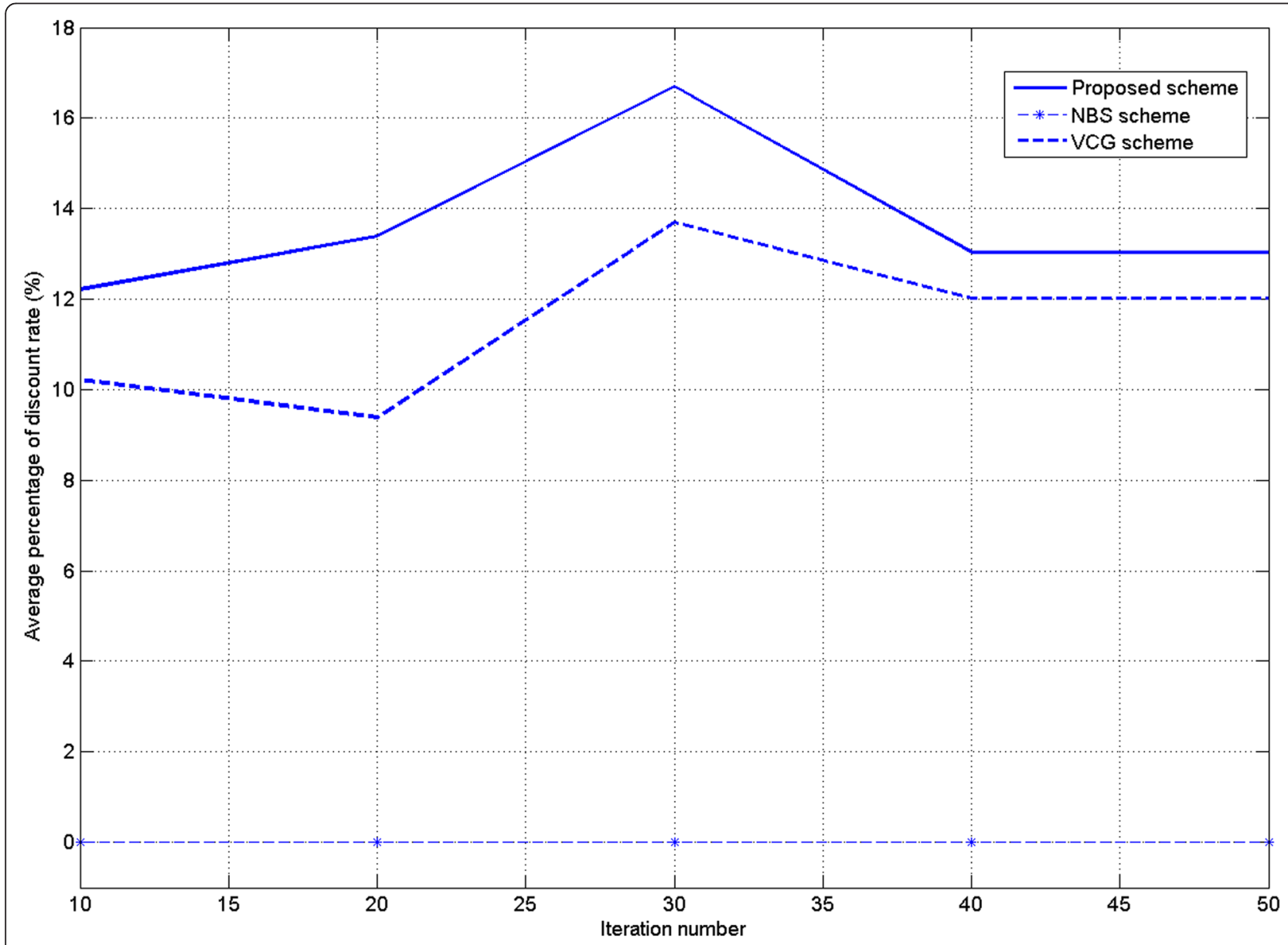

Fig. 5 loTMs' average percentage of discount rate 
on the game model. According to the VCG mechanism and Rubinstein bargaining solution, we make the IoT/ cellular system more efficient. The numerical results have shown the effectiveness of our proposed scheme and confirmed the feasibility of interactive game approach. For the future work, our game-based data offloading mechanism can be applied to many directions in various research areas and sheds light on different decision problems. For example, it would be useful to study the noncooperative game model under complete information.

\section{Competing interests}

The authors declare that they have no competing interests.

\section{Authors' contributions}

Y. Park and S. Kim are authors of this work and ES participated in the design of the study and performed the statistical analysis. All authors read and approved the final manuscript.

\section{Acknowledgements}

This research was supported by the Ministry of Science, ICT and Future Planning (MSIP), Korea, under the Information Technology Research Center (ITRC) support program (IITP-2015-H8501-15-1018) supervised by the Institute for Information and Communications Technology Promotion (IITP).

Received: 13 May 2015 Accepted: 15 June 2015

Published online: 16 July 2015

\section{References}

1. Q Wangl, T He, K-C Chen, J Wangl, B Ko, Y Linl, K-w Lee, Dynamic spectrum allocation under cognitive cell network for M2M applications, in Signals, Systems and Computers (ASILOMAR), 2012, pp. 596-600

2. G Lin, $\mathrm{G}$ losifidis, H Jianwei, L Tassiulas, Economics of mobile data offloading, in Computer Communications Workshops (INFOCOM WKSHPS), 2013, pp. 351-356

3. Z Sheng, S Yang, Y Yu, A Vasilakos, J McCann, K Leung, A survey on the IETF protocol suite for the internet of things: standards, challenges, and opportunities. IEEE Wireless Communications 20(6), 91-98 (2013)

4. Q Jing et al., Security of the Internet of things: perspectives and challenges. Wireless Networks 20(8), 2481-2501 (2014)

5. A Attar et al., A survey of security challenges in cognitive radio networks: solutions and future research directions. Proceedings of the IEEE 100(12), 3172-3186 (2012)

6. M Youssef et al., Routing metrics of cognitive radio networks: a survey. IEEE Communications Surveys and Tutorials 16(1), 92-109 (2014)

7. MA Khan et al., Game dynamics and cost of learning in heterogeneous $4 \mathrm{G}$ networks. IEEE Journal on Selected Areas in Communications 30(1), 198-213 (2012)

8. X Zhuo, W Gao, G Cao, S Hua, An incentive framework for cellular traffic offloading. IEEE Transactions on Mobile Computing 13(3), 541-555 (2014)

9. Y Park, S Kim, Bargaining-based smart grid pricing model for demand side management scheduling. ETRI Journal 37(1), 197-202 (2014)

10. B Sang-Seon et al., Computation of an equilibrium in spectrum markets for cognitive radio networks. IEEE Transactions on Computers 63(2), 304-316 (2014)

11. J Tigang et al., QoE-driven channel allocation schemes for multimedia transmission of priority-based secondary users over cognitive radio networks. IEEE Journal on Selected Areas in Communications 30(7), 1215-1224 (2012)

12. Z Hong et al., A framework for truthful online auctions in cloud computing with heterogeneous user demands, in IEEE INFOCOM, 2013, pp. 1510-1518

13. Z Feng et al., TRAC: truthful auction for location-aware collaborative sensing in mobile crowdsourcing, in IEEE INFOCOM, 2014, pp. 1231-1239

14. R Reza et al., MAPCloud: mobile applications on an elastic and scalable 2-tier cloud architecture, in Proceeding UCC '12 Proceedings of the 2012 IEEE/ACM Fifth International Conference on Utility and Cloud Computing, 2012, pp. 83-90
15. R Reza et al., MuSIC: mobility-aware optimal service allocation in mobile cloud computing, in 2013 IEEE Sixth International Conference on Cloud Computing (CLOUD), 2013, pp. 75-82

16. R Reza et al., Mobile cloud computing: a survey, state of art and future directions. MONET 19(2), 133-143 (2014)

17. F Xu et al., Managing performance overhead of virtual machines in cloud computing: a survey, state of the art, and future directions. Proceedings of the IEEE 102(1), 11-31 (2014)

18. D Panagiotis et al., Service configuration and traffic distribution in composite radio environments, in IEEE Transactions on Systems, Man, and Cybernetics, 2004, pp. 69-81

19. Y Zheng et al., A survey on trust management for Internet of things. Journal of Network and Computer Applications 42, 120-134 (2014)

20. L Gao, G losifidis, J Huang, D Li, Bargaining-based mobile data offloading. IEEE Journal on Selected Areas in Communications 32(6), 1114-1125 (2014)

21. Q Liu, G Feng, S Qin, Energy-efficient traffic offloading in macro-pico networks, in Wireless and Optical Communication Conference (WOCC), 2013, pp. 236-241

\section{Submit your manuscript to a SpringerOpen ${ }^{\odot}$ journal and benefit from:}

- Convenient online submission

- Rigorous peer review

- Immediate publication on acceptance

- Open access: articles freely available online

- High visibility within the field

- Retaining the copyright to your article

Submit your next manuscript at $\boldsymbol{\triangleright}$ springeropen.com 